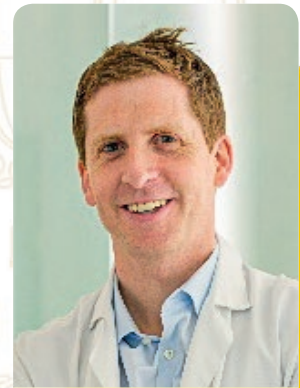

Prof. Dr. med.

Thomas Pilgrim.

(c) Schweizerische

Herzstiftung/

W. Tschan)

Forschungspreis der Schweizerischen Herzstiftung 2021

Der Herzklappenspezialist Prof. Dr. med. Thomas Pilgrim hat von der Schweizerischen Herzstiftung den Forschungspreis 2021 erhalten. Der am Berner Inselspital tätige Prof. Pilgrim hat in einer internationalen Studie die Sicherheit und Wirksamkeit von zwei grundlegend verschiedenen Klappensystemen verglichen. Das Forschungsresultat zeigt, dass das Einsetzen einer Herzklappe mittels eines Ballons grundsätzlich zu besseren Ergebnissen führt. Anhand von diesem Ergebnis können in Zukunft bessere Entscheide getroffen werden, welche Art der kathetergestützten Herzklappenimplantation vorgenommen werden soll. Prof. Pilgrim erhält den mit CHF 20000 dotierten Preis für diese Studie sowie für seinen Beitrag an ein Forschungsprojekt, welches sich mit Herzklappenfehlern bei Kindern und Jugendlichen in Nepal beschäftigt.

\section{Prix Luc Ciompi 2021}

La Dre Lilit Abrahamyan, du Service de psychiatrie générale du Centre hospitalier universitaire vaudois (CHUV), a reçu le Prix Luc Ciompi 2021 pour ses travaux visant à mieux comprendre et améliorer les traitements des psychoses schizophréniques. Dre Abrahamyan a exploré la relation entre la vie en milieu urbain et les psychoses; sujet qui constitue le corps de sa thèse doctorale. Son travail s'inscrit dans un projet interdisciplinaire financé par le Fonds national suisse de la recherche scientifique (FNS). La lauréate bénéficie d'une bourse de la re-

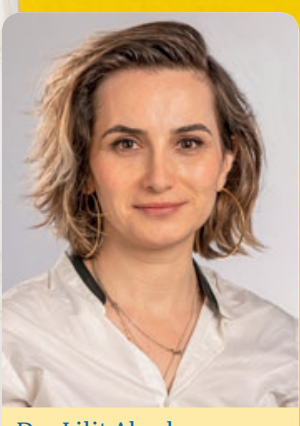

Dre Lilit Abrahamyan. (c CHUV) cherche financée par le Département de psychiatrie qui vise à promouvoir la relève académique.

Parrainé par le Prof. Luc Ciompi, ancien médecin-chef à l'Hôpital de Cery, le Prix Luc Ciompi est attribué tous les deux ans par la Société suisse de psychiatrie et psychothérapie (SSPP).
Dr. Deborah Rudin von der Universität Basel erhält den diesjährigen Young Scientist Award der Schweizerischen Gesellschaft für Klinische Pharmakologie und Toxikologie (SGKPT). Die SGKPT zeichnet Dr. Rudin für die von ihr verfasste PhD Arbeit «Toxicological and clinical investigations of metamizole-associated neutropenia» aus. Der Preis ist mit CHF 1000 dotiert.

\section{Young Scientist Award 2021}

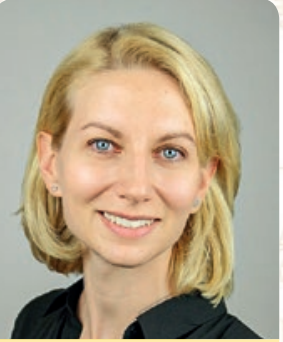

Dr. Deborah Rudin. (๔ Deborah Rudin)

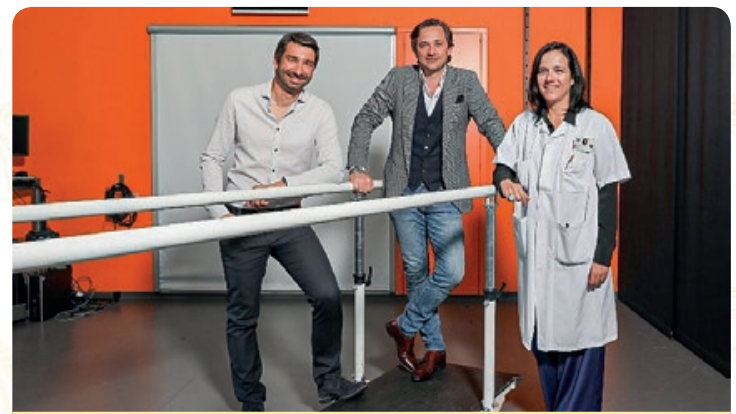

Prof. Grégoire Courtine (EPFL, au centre), entouré de Prof. Jocelyne Bloch (CHUV) et de Guillaume Charvet (CEA - Leti Clinatec). (๔ Alain Herzog)

\section{Prix scientifiques Leenaards 2021}

La Fondation Leenaards a décerné deux prix scientifiques à des groupes de recherche de l'Arc lémanique, pour un montant total de CHF 1,4 million. L'un des projets scientifiques primés, mené par le Prof. Grégoire Courtine, vise à étudier l'interface cerveau-moelle épinière afin de permettre aux paraplégiques de retrouver l'usage de leurs jambes. Ce projet a permis à neuf personnes de refaire l'expérience de la marche de manière quasi autonome, alors que leur condition les condamnait définitivement au fauteuil roulant. L'autre projet lauréat explore, sous l'égide de la Prof. Elisa Oricchio, la manière la plus naturelle possible de permettre au système immunitaire de reconnaître les cellules cancéreuses, dans le cadre de cancers lymphatiques. La découverte d'une mutation de la protéine Cathepsine S - qui permet aux cellules cancéreuses de passer inaperçues et donc de ne pas être attaquées par les anticorps - offre des perspectives thérapeutiques prometteuses dans la lutte contre le cancer.

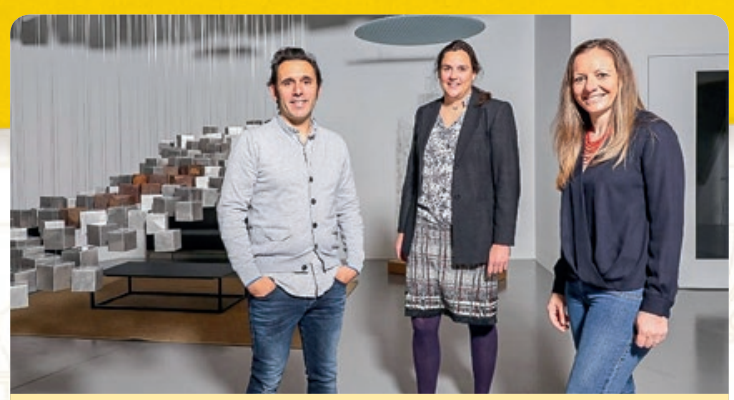

Prof. Elisa Oricchio (EPFL, à dr.), aux côtés de Prof. Caroline Arber (CHUV - UNIL - Ludwig Institute for Cancer Research) et de Prof. Bruno Correia (EPFL). (๔ Alain Herzog) 